\title{
Impacts of Beam Broadening and Earth Curvature on Storm-Scale 3D Variational Data Assimilation of Radial Velocity with Two Doppler Radars
}

\author{
GUOQING Ge \\ Center for Analysis and Prediction of Storms, and School of Meteorology, University of Oklahoma, Norman, Oklahoma \\ JiDONG GAO AND KEITH BREWSTER \\ Center for Analysis and Prediction of Storms, University of Oklahoma, Norman, Oklahoma \\ MiNG XUE \\ Center for Analysis and Prediction of Storms, and School of Meteorology, University of Oklahoma, Norman, Oklahoma
}

(Manuscript received 13 July 2009, in final form 7 December 2009)

\begin{abstract}
The radar ray path and beam broadening equations are important for assimilation of radar data into numerical weather prediction (NWP) models. They can be used to determine the physical location of each radar measurement and to properly map the atmospheric state variables from the model grid to the radar measurement space as part of the forward observation operators. Historically, different degrees of approximations have been made with these equations; however, no systematic evaluation of their impact exists, at least in the context of variational data assimilation. This study examines the effects of simplifying ray path and ray broadening calculations on the radar data assimilation in a 3D variational data assimilation (3DVAR) system. Several groups of Observational System Simulation Experiments (OSSEs) are performed to test the impact of these equations to radar data assimilation with an idealized tornadic thunderstorm case. This study shows that the errors caused by simplifications vary with the distance between the analyzed storm and the radar. For single time level wind analysis, as the surface range increases, the impact of beam broadening on analyzed wind field becomes evident and can cause relatively large error for distances beyond $150 \mathrm{~km}$. The impact of the earth's curvature is more significant, even for distances beyond $60 \mathrm{~km}$, because it places the data at the wrong vertical location. The impact of refractive index gradient is also tested. It is shown that the variations of refractive index gradient have a very small impact on the wind analysis results.

Two time series of 1-h-long data assimilation experiments are further conducted to illustrate the impact of the beam broadening and earth curvature on all retrieved model variables. It is shown that all model variables can be retrieved to some degrees in all data assimilation experiments. Similar to the wind analysis experiments, the impacts of both factors are not obvious when radars are relatively close to the storm. When the radars are far from the storm (especially beyond $150 \mathrm{~km}$ ), overlooking beam broadening degrades the accuracy of assimilation results slightly, whereas ignoring the earth's curvature leads to significant errors.
\end{abstract}

\section{Introduction}

The operational Weather Surveillance Radar-1988 Doppler (WSR-88D) Next Generation Weather Radar (NEXRAD) system is an important tool for real-time detection and warning of hazardous weather (Crum and

Corresponding author address: Guoqing Ge, Center for Analysis and Prediction of Storms, and School of Meteorology, University of Oklahoma, 120 David L. Boren Blvd., Norman, OK 73072.

E-mail: geguoqing@ou.edu
Alberty 1993; Crum et al. 1998; Serafin and Wilson 2000). It is also an essential observing system for initializing nonhydrostatic, storm-resolving (i.e., horizontal grid spacing on the order of $1 \mathrm{~km}$ ) numerical weather prediction (NWP) models (e.g., Lilly 1990; Droegemeier 1990, 1997). To assimilate these radar data into NWP models, it is necessary to accurately determine the spatial locations of individual radar measurements. Because the propagation path of the electromagnetic waves can be affected by the refractivity of the atmosphere, the propagation path or the ray path is usually not a straight line. 
A suitable ray path equation is therefore needed. The local direction of the ray path also affects the radial velocity forward operator that projects the Cartesian velocity components on the model grid to the local radial direction in data assimilation systems.

Most early radar data assimilation studies used relatively simple ray path equations in the forward operator formulation, which are based on the Cartesian geometry, essentially assuming a flat earth (e.g., Sun et al. 1991; Qiu and Xu 1992, 1996; Xu et al. 1995; Sun and Crook 1997, 1998; Gao et al. 1998, 2004; Xu et al. 2001; Weygandt et al. 2002a,b; Shapiro et al. 2003). Brewster (2003) applied complete ray path equations into the Advanced Regional Prediction System (ARPS; Xue et al. 2000, 2001, 2003) Data Assimilation System (ADAS) and phase correction technique. Similar ray path equations were applied into the 3.5-dimensional variational data assimilation (3.5DVAR) system (Gu et al. 2001; Zhao et al. 2006) developed for the Coupled Ocean-Atmosphere Mesoscale Prediction System (Hodur 1997). However, these previous studies did not investigate the impact of the ray path equations on the radar data assimilation systems. Gao et al. (2006, hereafter Gao06) have shown that using simplified radar ray path equations introduces errors that are significant for ranges beyond $30 \mathrm{~km}$. In that paper, a set of four-thirds earth radius ray path equations is recommended, especially at low elevation angles. However, Gao06 mainly addresses the error in physical location of individual radar measurement. It is also of interest to study how, and to what extent, the neglecting of earth curvature will affect the results of storm-scale radar data assimilation.

To compute most accurately the model counterpart of radial wind, one must integrate over all possible model grid points within the radar beam main lobe, which broadens with range. Most radar data assimilation studies do not consider this beam broadening effect. Wood and Brown (1997) introduced a power-gain weighted average in the radar forward observation operator in their study on the effects of radar sampling on velocity signatures of mesocylones and tornadoes. Sun and Crook (2001) incorporated a similar beam broadening equation in their 4DVAR radar analysis system. Salonen (2002) approximated the beam broadening effect with a Gaussian function (Probert-Jones 1962) in the vertical direction and demonstrated slightly positive impact on radar analysis using the High Resolution Limited Area Model (HIRLAM) 3DVAR system. Xue et al. (2006) and Tong (2006) used a power-gain-based sampling in vertical direction to compute the model counterpart of radial velocity in their ensemble Kalman filter (EnKF) work. All these treatments are more reasonable because they are close to the nature of the radar measurement. Caumont and Ducrocq (2008) showed that neglecting the beam broadening could cause large errors at distant gates in the simulation of radar data. However, a detailed study of the effect of beam broadening in storm-scale data analysis and assimilation has not yet been investigated.

In this study, the effect of earth curvature and beam broadening in radar data assimilation is investigated using an idealized supercell tornadic thunderstorm. The ARPS 3DVAR system, described in Gao et al. (2002, 2004) and Hu et al. (2006b), is used for this purpose. The ARPS 3DVAR system is capable of analyzing radar radial velocity data along with conventional observations. It is usually used together with the cloud analysis system to initialize hydrometeor-related variables and provide a latent heating adjustment. For simplicity in studying the radial velocity effects, in this paper only the simulated radial winds derived from an idealized thunderstorm are used and the cloud analysis is not used. In the ARPS 3DVAR system, the mass continuity weak constraint is included in the cost function that serves to link three wind components together and helps to improve wind analysis.

This paper is organized as follows: In sections 2 and 3, we will briefly introduce the radar forward observation operator and the ARPS 3DVAR system, respectively. In section 4 , the model configuration and experiment design are discussed. The results are presented in section 5 , and a summary and discussion are in section 6 .

\section{The radar forward observation operator}

Under the assumption that the refractivity is a function only of height above mean sea level, Doviak and Zrnic (1993) present a formulation that expresses the ray path in terms of a path following a curve of a sphere of radius,

$$
a_{e}=\frac{a}{1+a(d n / d h)}=k_{e} a,
$$

where $a$ is the earth's radius, $k_{e}$ is a multiplier that is dependent on the vertical gradient of refractive index of air $d n / d h, h$ is the height above the radar altitude, and $n$ is the refractive index of air. The assumptions under which Eq. (1) is reached also include the following: 1) the radar ray is launched at a low elevation angle, which is usually the case with weather radars; 2) the refractive index $n$ is close to 1 ; 3) $h \ll a$; and 4) $d h / d s \ll 1$, where $s$ is the surface range (distance along the earth's surface).

The refractive index of air $n$ is a function of its temperature, pressure, and humidity. It is convenient to use the quantity $N$, which is called radio refractivity, instead of $n ; N$ represents the departure of $n$ from unity in parts per million and its variations can be considered more conveniently; $N$ has a value of about 300 (at the surface). 
Usually, $N$ is taken, subject to certain assumptions, as (Bean and Dutton 1968)

$$
N=(n-1) \times 10^{6}=77.6 P / T+3.73 \times 10^{5} e T^{-2},
$$

where $P$ is air pressure in hectopascals (including water vapor pressure), $e$ is water vapor pressure in hectopascals, and $T$ is air temperature in kelvins. In Eq. (2), the first term on the right-hand side is known as the dry term, and the second term is the moist term. The value of $N$ can be computed from measurement of $P, T$, and $e$. If $h$ is limited to the lowest $20 \mathrm{~km}$ of the atmosphere and $d n / d h$ is $-1 /(4 a)$ in the lower atmosphere, $k_{e}$ will be equal to $4 / 3$ (Doviak and Zrnić 1993). This is often referred to as the "four-thirds earth radius model."

The following two equations relate $h$ and the surface range $s$ (distance along the earth's surface) to radarmeasurable parameters, the slant path $r$, and radar elevation angle $\theta_{e}$ (Doviak and Zrnić 1993):

$$
\begin{aligned}
& s=k_{e} a \sin ^{-1}\left(\frac{r \cos \theta_{e}}{k_{e} a+h}\right) \text { and } \\
& h=\left[r^{2}+\left(k_{e} a\right)^{2}+2 r k_{e} a \sin \theta_{e}\right]^{1 / 2}-k_{e} a .
\end{aligned}
$$

To consider the curvature of the earth, the radar forward observation operator can be written as the following equation:

$$
\begin{aligned}
v_{r}^{*}= & u \cos \theta_{e}^{\prime} \sin \phi+v \cos \theta_{e}^{\prime} \cos \phi \\
& +\left(w-w_{t}\right) \sin \theta_{e}^{\prime},
\end{aligned}
$$

where $\phi$ is radar azimuth angle, $w_{t}$ is the terminal velocity of precipitation, and $\theta_{e}^{\prime}$ includes the effect of the curvature of the earth as

$$
\theta_{e}^{\prime}=\theta_{e}+\tan ^{-1}\left[\frac{\left(r \cos \theta_{e}\right)}{\left(k_{e} a+r \sin \theta_{e}\right)}\right] .
$$

In this study, only the effect of beam broadening in the vertical direction is considered. The reason is as follows: In storm-scale NWP, the horizontal resolution is normally between 1 and $3 \mathrm{~km}$ and a $1^{\circ}$ half-power beamwidth will measure about $3490 \mathrm{~m}$ at a surface range of $200 \mathrm{~km}$. So, a beam lobe at a surface range of $200 \mathrm{~km}$ and gate spacing less than $1 \mathrm{~km}$ will enclose only 1-3 horizontal grid points, even at $1-\mathrm{km}$ grid spacing, which we judge to be too few to have a material difference. However, the vertical resolution of NWP models typically ranges from 20 to $500 \mathrm{~m}$ and a beam lobe at a range of $200 \mathrm{~km}$ can span more than seven vertical grid points, much greater than the two grid points that might be used to compute the model counterpart of radial wind with linear interpolation.
At the same time, the height of the lowest ray above the ground will increase rapidly with range (Gao06). At a surface range of $100 \mathrm{~km}$, the height of the center of a $0.5^{\circ}$ ray above the ground is about $1.5 \mathrm{~km}$, and at $200 \mathrm{~km}$ it is about $4 \mathrm{~km}$. So, there may be little information observed of the boundary layer, especially far from the radar. Considering beam broadening in the radar forward observation operator may also spread information below the center of the lowest ray.

Following Rihan et al. (2008), the observation operator for mapping data from multiple vertical model levels onto elevation angles is formulated as

$$
V_{r, e}=H_{e}\left(V_{r}\right)=\frac{\left(\sum G V_{r} \Delta z\right)}{\left(\sum G \Delta z\right)},
$$

where $V_{r, e}$ is the radial velocity on an elevation angle, $H_{e}$ is the radar forward observation operator, $V_{r}$ is the model counterpart of radial velocity, and $\Delta_{z}$ is the vertical model grid spacing. Here, $G$ describes the two-way power-gain distribution within the radar beam and is formulated as $G=e^{-4 \ln 4 \alpha^{2} / \beta^{2}}$ (Wood and Brown 1997), with $\alpha$ as the distance from the center of the radar beam in radians and $\beta$ as the $1^{\circ}$ beamwidth. The summation is over vertical model grid points enclosed by the halfpower beam lobe.

\section{The ARPS 3DVAR system}

Following Gao et al. (2004), the standard cost function of 3DVAR can be written as

$$
\begin{aligned}
J(\mathbf{x})= & \frac{1}{2}\left(\mathbf{x}-\mathbf{x}^{b}\right)^{\mathrm{T}} \mathbf{B}^{-1}\left(\mathbf{x}-\mathbf{x}^{b}\right) \\
& +\frac{1}{2}\left[H(\mathbf{x})-\mathbf{y}^{o}\right]^{\mathrm{T}} \mathbf{R}^{-1}\left[H(\mathbf{x})-\mathbf{y}^{o}\right]+J_{c}(\mathbf{x}),
\end{aligned}
$$

where the first term on the right-hand side measures the departure of the analysis vector $\mathbf{x}$ from the background vector $\mathbf{x}^{b}$ weighted by the inverse of the background error covariance matrix $\mathbf{B}$. In the current ARPS 3DVAR system, the analysis vector $\mathbf{x}$ contains the three wind components $u, v$, and $w$; potential temperature $\theta$, pressure $p$, and water vapor mixing ratio $q_{v}$. The second observation term measures the departure of the analysis from the observation vector $\mathbf{y}^{o}$. In this study, $\mathbf{y}^{o}$ only includes radar radial velocity data. The analysis is projected to the observation space by the forward operator $H$, which is defined by Eqs. (1)-(7) and an interpolation operator from model grid points to radar observation 
locations. The observation term is weighted by the inverse of observation error covariance matrix $\mathbf{R}$ that includes both instrument and representativeness errors. Because only radial velocity data are used in the analysis system and there are no cross correlations between variables in the $\mathbf{B}$ matrix, only wind components will be updated during the minimization process. Term $J_{c}(\mathbf{x})$ in Eq. (8) represents dynamic or equation constraints.

By defining $\sqrt{\mathbf{B} \mathbf{v}}=\left(\mathbf{x}-\mathbf{x}^{b}\right)$, the cost function is changed into incremental form,

$$
\begin{aligned}
J_{\text {inc }}(\mathbf{v})= & \frac{1}{2} \mathbf{v}^{\mathrm{T}} \mathbf{v} \\
& +\frac{1}{2}\left(\mathbf{H B}^{1 / 2} \mathbf{v}-\mathbf{d}\right)^{\mathrm{T}} \mathbf{R}^{-1}\left(\mathbf{H} \mathbf{B}^{1 / 2} \mathbf{v}-\mathbf{d}\right)+J_{c}(\mathbf{v}),
\end{aligned}
$$

where $\mathbf{H}$ is the linearized version of $H$ and $\mathbf{d} \equiv \mathbf{y}^{o}-H\left(\mathbf{x}^{b}\right)$. In the current version of ARPS 3DVAR system, the spatial covariances for background error are modeled by a recursive filter (Purser et al. 2003a,b). The corresponding covariance matrix $\mathbf{R}$ is diagonal, and its diagonal elements are specified according to the estimated observation errors $\left(1 \mathrm{~m} \mathrm{~s}^{-1}\right.$ in this study).

In the ARPS 3DVAR, the mass continuity equation is imposed as a weak constraint. This constraint builds up the relationship among the three wind components. Gao et al. $(1999 ; 2004)$ found that this constraint is very effective in producing suitable analyses of vertical velocity. When a stretched grid strategy is used in the vertical direction, a special treatment (Hu et al. 2006a,b), which assigns different weighting coefficients in horizontal and vertical direction, is needed to apply this constraint. More recently, the modified ARPS model equations are included as weak constraints in the 3DVAR scheme. These newly introduced constraints couple the wind components with thermodynamic variables (Ge and Gao 2007). In this study, for simplicity, only the mass continuity constraint is included.

\section{Experimental design}

In this study, we evaluate the impact of beam broadening and earth curvature on data assimilation system using simulated data. Such simulation experiments are usually referred to as observing system simulation experiments (OSSEs). The ARPS model is used in a 3D cloud model mode. The 20 May 1977 Del City, Oklahoma, tornadic supercell storm is used to conduct several series of experiments. This storm has been thoroughly studied by multiple Doppler analysis and numerical simulation (Ray et al. 1981; Klemp et al. 1981; Klemp and Rotunno 1983).

The model is configured as follows: $67 \times 67 \times 35$ grid points and $1 \mathrm{~km} \times 1 \mathrm{~km} \times 0.5 \mathrm{~km}$ grid intervals for the $x, y$, and $z$ directions, respectively, so as to establish a physical domain of $64 \mathrm{~km} \times 64 \mathrm{~km} \times 16 \mathrm{~km}$. The simulation starts with a modified sounding (as in Klemp et al. 1981), which favors the development of a supercell thunderstorm. The thermal bubble has a $4-\mathrm{K}$ perturbation and is centered at $x=48 \mathrm{~km}, y=16 \mathrm{~km}$, and $z=$ $1.5 \mathrm{~km}$ with the bottom-left corner of the domain as the origin. The radius of the bubble is $10 \mathrm{~km}$ in the $x$ and $y$ directions and $1.5 \mathrm{~km}$ in the $z$ direction. The threecategory ice microphysical scheme of Lin et al. (1983) is used together with a 1.5 -order turbulent kinetic energy subgrid parameterization. Open boundary conditions are used for the lateral boundaries and rigid wall conditions for the top and bottom boundaries. An upperlevel Rayleigh damping layer is also included to inhibit wave reflection from the top of the model.

The simulation runs for $3 \mathrm{~h}$. The initial convective cell strengthens over the first $20 \mathrm{~min}$ and begins to split into two cells at around $1 \mathrm{~h}$. To keep the right-moving storm near the center of the model domain, a mean storm speed $\left(U=3 \mathrm{~m} \mathrm{~s}^{-1}, V=14 \mathrm{~m} \mathrm{~s}^{-1}\right)$ is subtracted from the sounding. At about $2 \mathrm{~h}$ into the simulation, the right mover is still near the center of the domain as expected and the left mover is located at the northwest corner. Figures 1a and 2a show horizontal and vertical cross sections of simulated wind and vertical velocity at $2 \mathrm{~h}$, respectively (vertical cross section is plotted through line A-B in Fig. 1a). A strong rotating updraft (with maximum vertical velocity exceeding $29 \mathrm{~m} \mathrm{~s}^{-1}$ ) and associated low-level downdraft are evident near the center of the domain. The updraft tilts eastward in the upper part of the troposphere. The evolution of the simulated storm is qualitatively similar to that described by Klemp et al. (1981). After $2 \mathrm{~h}$, the major storm gradually moves a little bit toward the southeastern corner of the model domain and remains a very strong supercell structure until the end of simulation at $3 \mathrm{~h}$ (Figs. 7a-c).

Four series of pseudo-radar radial observations from two Doppler radars are obtained by sampling the evolution of this simulated storm every 5 min from 2 to $3 \mathrm{~h}$ using radar forward operators expressed in Eqs. (1)-(7). The first series of simulated data are obtained from the simulated wind field fixed at $t=2 \mathrm{~h}$, as a function of various radar locations. Of the two radars, one is put at $x=33 \mathrm{~km}$ relative to the origin of model domain (bottom-left corner), whereas its $y$ coordinate is varied in increments of $10 \mathrm{~km}$ from $y=-190 \mathrm{~km}$ to $y=10 \mathrm{~km}$. A second radar is set at position $y=25 \mathrm{~km}$, whereas its $x$ coordinate is varied from $x=0 \mathrm{~km}$ to $x=-200 \mathrm{~km}$ in intervals of $10 \mathrm{~km}$. In this way, we are able to test the impact of the beam broadening and the earth curvature as a function of distance from the center of the storm ranging from about 20 to $220 \mathrm{~km}$. The center of the storm 

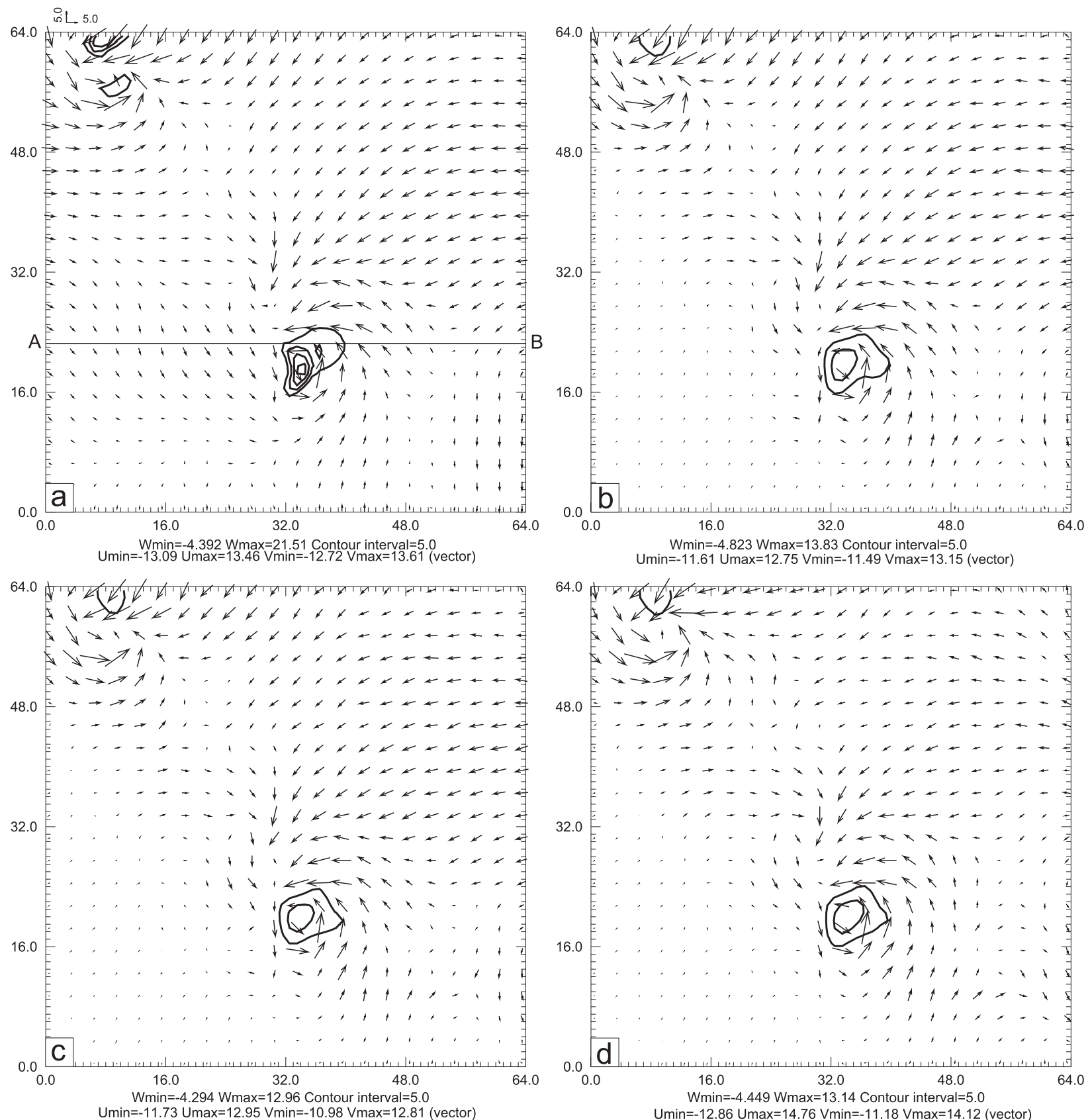

FIG. 1. Perturbation horizontal winds (vectors; $\mathrm{m} \mathrm{s}^{-1}$ ) and vertical velocity $w$ (contours; $\mathrm{m} \mathrm{s}^{-1}$ ) at $t=120 \mathrm{~min}$ and $3.5 \mathrm{~km} \mathrm{AGL} \mathrm{for} \mathrm{(a)} \mathrm{truth}$ simulation, (b) CNTL1_60, (c) NoBB1_60, and (d) NoCV1_60. The $w$ contour starts from $5 \mathrm{~m} \mathrm{~s}^{-1}$ with an interval of $5 \mathrm{~m} \mathrm{~s}$.

is estimated to be $(32.5 \mathrm{~km}, 22.5 \mathrm{~km})$. The second series of pseudo-observations are sampled in a similar way to the first one, except that the refractive index gradient $d n / d h$ is no longer $-1 /(4 a)$ (about $-39.2 \times 10^{-6} \mathrm{~km}^{-1}$ ) in Eq. (1) for the four-thirds earth radius model. Instead, the $d n / d h$ takes the values of $-10 \times 10^{-6},-70 \times 10^{-6}$, $-100 \times 10^{-6}$, and $-130 \times 10^{-6} \mathrm{~km}^{-1}$, respectively, representing most possible cases in the atmosphere.
The third series of radial velocity observations are obtained every 5 min from model simulation between 2 and $3 \mathrm{~h}$ using the same forward operator, but two radars are at fixed locations $(33 \mathrm{~km},-40 \mathrm{~km})$ and $(-30 \mathrm{~km}$, $25 \mathrm{~km}$ ), respectively. In this case, the surface range between the storm center and either of the radars is about $60 \mathrm{~km}$. The fourth series of pseudo-observations are sampled in a similar way to the third series, for two radars at 

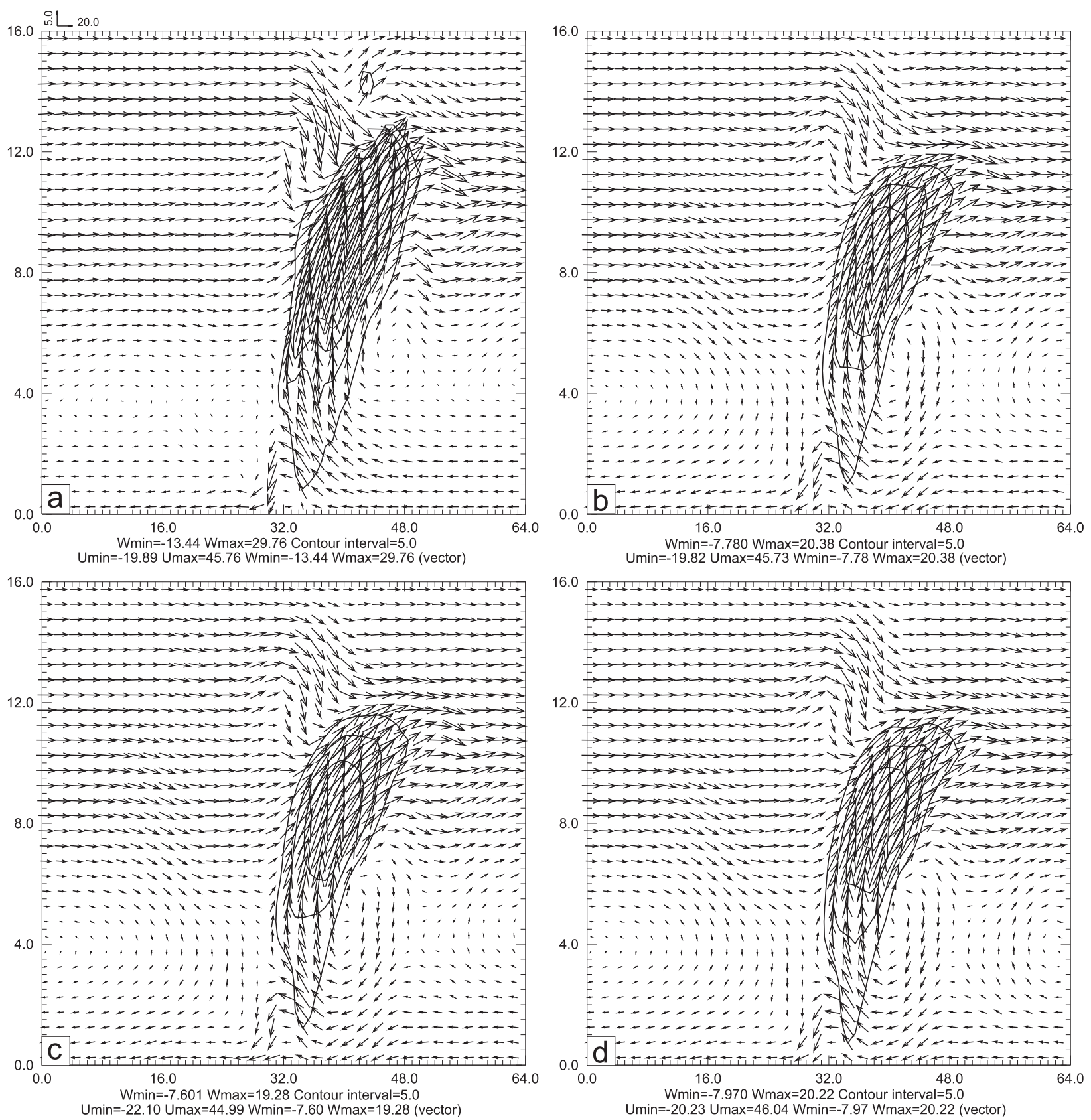

FIG. 2. Total $u-w$ wind vectors and vertical velocity (contours) of the 20 May 1977 supercell storm at $t=120 \mathrm{~min}$ and $y=22.5 \mathrm{~km}$ (along the line A-B in Fig. 1a) for (a) truth simulation, (b) CNTL1_60, (c) NoBB1_60, and (d) NoCV1_60.

fixed locations $(33 \mathrm{~km},-130 \mathrm{~km})$ and $(-120 \mathrm{~km}, 25 \mathrm{~km})$. In this case, the distance between the storm center and either of the radars is about $150 \mathrm{~km}$.

The elapsed times for the radars to obtain the volume scans are neglected; thus, we assume that the radial wind observations are simultaneous. For simplicity, the two radars will cover the entire horizontal physical grids (i.e., $64 \mathrm{~km} \times 64 \mathrm{~km}$ ), which assumes that the radars sweep almost continuously in the horizontal direction. The elevation angles are $0.5^{\circ}, 0.9^{\circ}, 1.3^{\circ}, 2.4^{\circ}, 3.1^{\circ}, 4.0^{\circ}, 5.1^{\circ}$, $6.4^{\circ}, 7.5^{\circ}, 8.7^{\circ}, 10.0^{\circ}, 12.0^{\circ}, 16.7^{\circ}$, and $19.5^{\circ}$ [same as the WSR-88D convective precipitation volume coverage pattern (VCP) 11]. The simulated data are only specified in precipitation regions (where reflectivity is greater than $0 \mathrm{dBZ}$ ). To simulate the radar measurement statistical error, $1 \mathrm{~m} \mathrm{~s}^{-1}$ random error (white noise) is added to the radial velocities in the pseudo-observation data. 
TABLE 1. List of data analysis/assimilation experiments.

\begin{tabular}{|c|c|c|}
\hline Name* & Radar distance & Description \\
\hline CNTL1_xxx & $20-220 \mathrm{~km}$ at an & One-time analyses \\
\hline NoBB1_xxx & interval of $10 \mathrm{~km}$ & at $t=2 \mathrm{~h}(21$ \\
\hline NoCV1_xxx & $\begin{array}{l}\text { (xxx is the radar } \\
\text { distance in } \mathrm{km} \text { ) }\end{array}$ & $\begin{array}{l}\text { experiments } \\
\text { for each type) }\end{array}$ \\
\hline CNTLM_60 & $60 \mathrm{~km}$ & 1-h assimilation \\
\hline NoBBM 60 & & from $t=2$ to $3 \mathrm{~h}$ \\
\hline NoCVM_60 & & at 5-min intervals \\
\hline CNTLM_150 & $150 \mathrm{~km}$ & \\
\hline \multicolumn{3}{|l|}{ NoBBM_150 } \\
\hline \multicolumn{3}{|l|}{ NoCVM_150 } \\
\hline DnDhxxx & $\begin{array}{l}20-220 \mathrm{~km} \text { at an } \\
\text { interval of } \\
10 \mathrm{~km}(\mathrm{xxx} \text { is } \\
\text { the } d n / d h \text { value })\end{array}$ & $\begin{array}{l}\text { One-time analyses } \\
\text { at } t=2 \mathrm{~h}(21 \\
\text { experiments for } \\
\text { each DnDhxxx } \\
\text { experiment) }\end{array}$ \\
\hline
\end{tabular}

* CNTL means both the effects of beam broadening and earth curvature are considered, NoBB means the effects of beam broadening are neglected, and NoCV means the effects of earth curvature are neglected.

Corresponding to the first series of radial wind observations, three categories, with 21 experiments for each category, of data analysis experiments (see Table 1, which lists all experiments) will be conducted at $t=2 \mathrm{~h}$ with varied surface ranges between radar location and storm center. In the first category of experiments, both the effect of beam broadening and the effects of earth curvature are considered using the radar forward observation operator as defined in Eqs. (1)-(7). They will be referred as CNTL1 experiments (label 1 means at single time level). In the second category of experiments, the effect of beam broadening is not considered, and Eq. (7) will be replaced with a simple trilinear interpolation scheme. It will be referred to as NoBB1 experiments. In the third category of experiments, the effect of earth curvature will not be considered, and Eqs. (3)-(6) will be replaced with the commonly used Cartesian radar forward operator (Gao et al. 1999). It will be referred as NoCV1 experiments. The distance between the storm and the radar varies from 20 to $220 \mathrm{~km}$ at an interval of $10 \mathrm{~km}$ for both radars. Therefore, each individual experiment will be referred to by its category name followed by the distance in kilometers, as described above (e.g., CNTL1_60, NoBB1_60, NoCV1_60, etc). Corresponding to the second series of pseudo-observations, four categories, with 21 experiments for each category, of data analysis experiments are performed (see Table 1). The settings are similar to that in CNTL1 experiments, except that the refractive index gradient $d n / d h$ is no longer $-1 /(4 a)$. The four categories of experiments are named DnDh10, DnDh-70, DnDh-100, and DnDh-130, respectively, according to the value of $d n / d h$ used.
Corresponding to the third series of pseudoobservations, three intermittent data assimilation experiments (see Table 1) are performed with an interval of $5 \mathrm{~min}$ and a window covering $t=2 \mathrm{~h}$ to $t=3 \mathrm{~h}$ of the model simulation. For these three experiments, the distance from the radar to the storm center is about $60 \mathrm{~km}$ when the data assimilation experiments begin. These three experiments are referred as CNTLM_60, NoBBM_60, and NoCVM_60 experiments with similar literal meaning as the above (where the label $\mathrm{M}$ is added to denote multiple time levels). Corresponding to the fourth series of pseudo-observations, three more intermittent data assimilation experiments (see Table 1) are performed. The setting is same as above, but the distance between radar location and storm center is changed to $150 \mathrm{~km}$ at the beginning of data assimilation. Similarly, these three more experiments are named CNTLM_150, NoBBM_150, and NoCVM_150. These six experiments are designed to assess the impact of the beam broadening and the earth curvature on radar data assimilation over a data assimilation window while radar sites are near or far from a storm. There are 13 assimilation cycles with 5-min interval in these six experiments. The ARPS 3DVAR system is used to obtain the model initial condition first, and then the ARPS system runs for a 5-min forecast starting from this initial analysis. This intermittent assimilation cycle is applied every $5 \mathrm{~min}$ until the end of assimilation period.

To compare the accuracy of the analysis from different experiments, the RMS error statistics of the horizontal wind components $V_{h}$ and scalar model variables $s$ between the experiments and the truth simulation run are computed using the following equations:

RMS_Vh $=\sqrt{\frac{\sum_{i=1}^{N}\left(u-u_{\text {simu }}\right)_{i}^{2}+\sum_{i=1}^{N}\left(v-v_{\text {simu }}\right)_{i}^{2}}{2 N}}$ and

$$
\operatorname{RMS} \_s_{-}=\sqrt{\frac{\sum_{i=1}^{N}\left(s-s_{\text {simu }}\right)_{i}^{2}}{N}}
$$

where $N$ is the total number of three-dimensional grid points used in the calculation and the subscript simu stands for the data from the simulation run. The computation of the RMS error statistics is only done over model grid points where the reflectivity (estimated from the local hydrometeor mixing ratios) of the simulation run is greater than $5 \mathrm{~dB} Z$. 

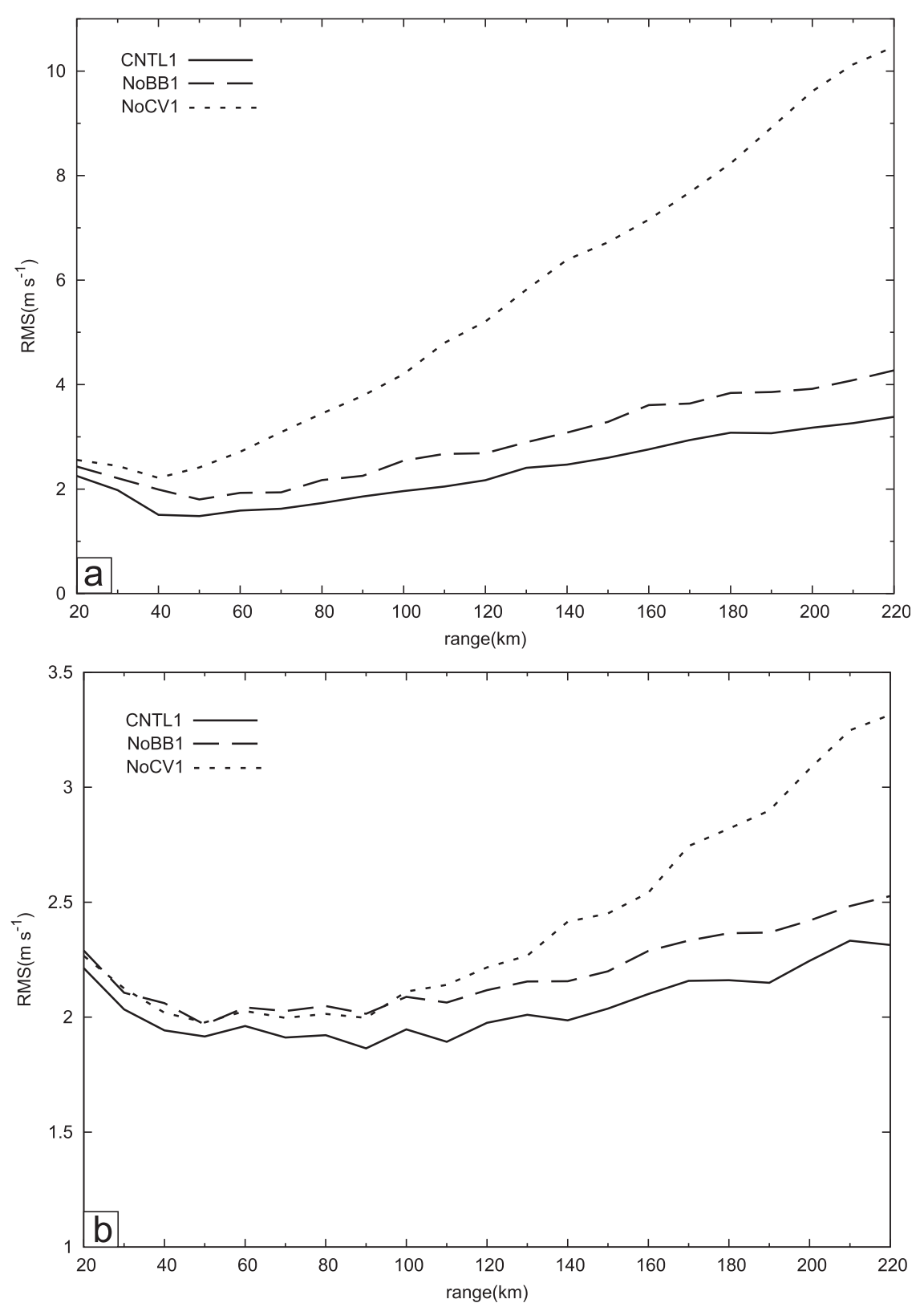

FIG. 3. The variation of RMS errors with the distance between the center of the storm and radar locations for (a) horizontal wind and (b) vertical velocity. The solid lines are for CNTL1 experiments, the dashed lines are for the NoBB1 experiments, and the dotted lines are for the NoCV1 experiments.

\section{Results of experiments}

a. The impact on $3 D V A R$ wind analysis at $\mathrm{t}=2 \mathrm{~h}$ time level

As stated above, the purpose of first series of experiments is to test the impact of beam broadening and earth curvature on 3DVAR wind analysis at a single time level. The variations of RMS errors for NoBB1 and NoCV1 are plotted in Fig. 3 along with that for CNTL1. The hori- zontal section at $z=3.5 \mathrm{~km}$ AGL and the vertical cross section at $y=22.5 \mathrm{~km}$ of wind fields for the truth simulation, CNTL1_60, NoBB1_60, NoCV1_60 (Figs. 1 and 2), and CNTL1_150, NoBB1_150, NoCV1_150 are plotted in Figs. 4 and 5.

We first discuss the impact of beam broadening. The RMS error of the horizontal winds and the vertical velocities plotted as a function of the distance for both CNTL1 (solid lines) and NoBB1 (dashed lines) experiments are 

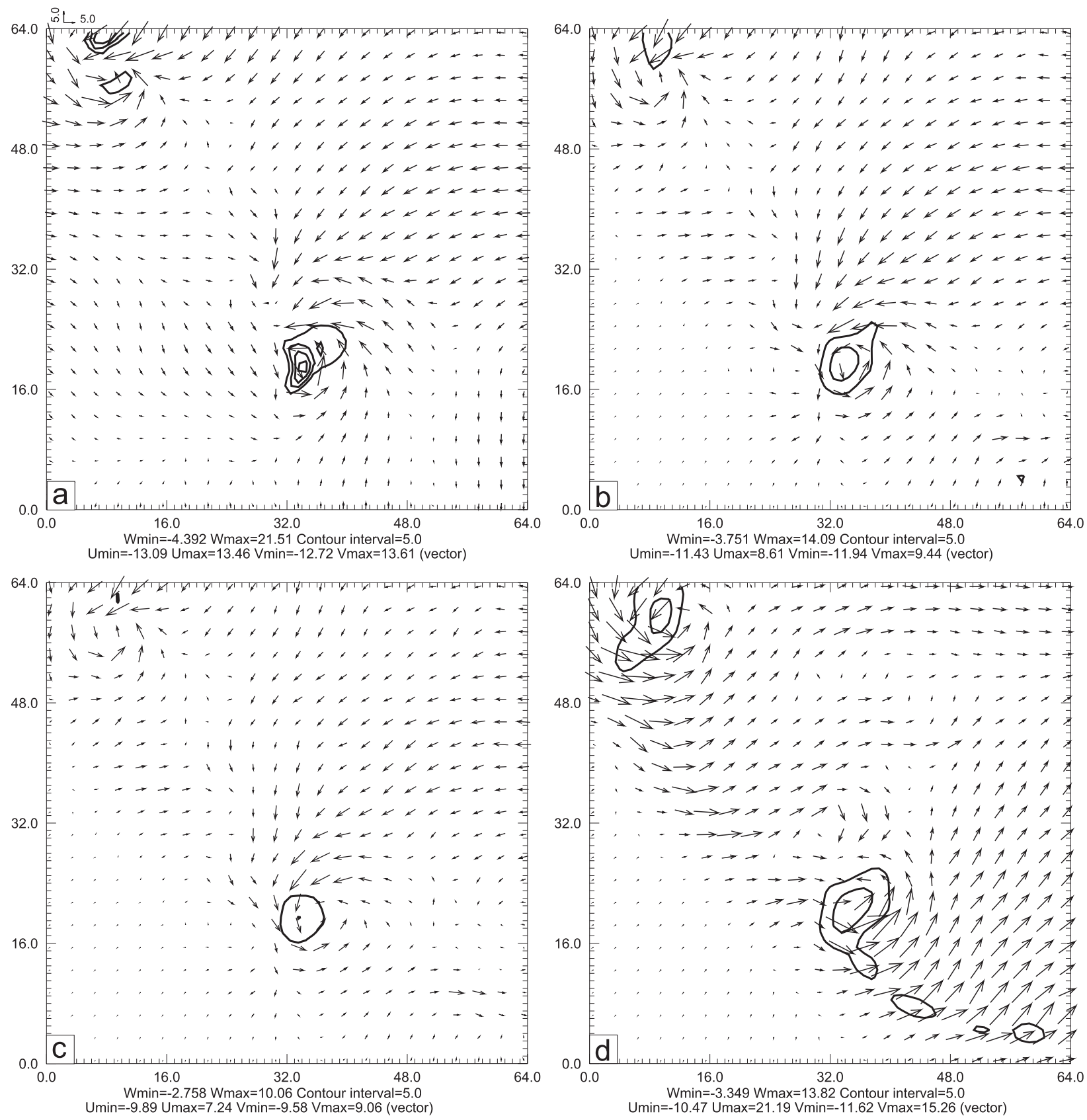

FIG. 4. As in Fig. 1, but for (a) truth simulation,

(b) CNTL1_150, (c) NoBB1_150, and (d) NoCV1_150.

shown in Fig. 3. It is found that the RMS error differences for both horizontal winds and vertical velocities between these 21 CNTL1 experiments and their corresponding NoBB1 experiments gradually increase as the distance between the storm center and radar locations increase. These differences are less than $0.35 \mathrm{~m} \mathrm{~s}^{-1}$ for horizontal winds and less than $0.1 \mathrm{~m} \mathrm{~s}^{-1}$ for vertical velocities within the range of $60 \mathrm{~km}$. Beyond $60 \mathrm{~km}$, the differences for horizontal winds become more noticeable as the range increases, reaching over $1 \mathrm{~m} \mathrm{~s}^{-1}$ at the range of $220 \mathrm{~km}$, whereas the difference for vertical velocity shows little change. This means that additional error due to the neglect of beam broadening is gradually introduced in NoBB1 experiments.

The variation in the RMS errors for horizontal winds and vertical velocities as a function of distance for experiment NoCV1 is also plotted in Fig. 3 in dotted lines. It is easily identified that the neglecting of the earth curvature can lead to very large RMS errors in the analysis of horizontal winds, especially beyond $60 \mathrm{~km}$. It 

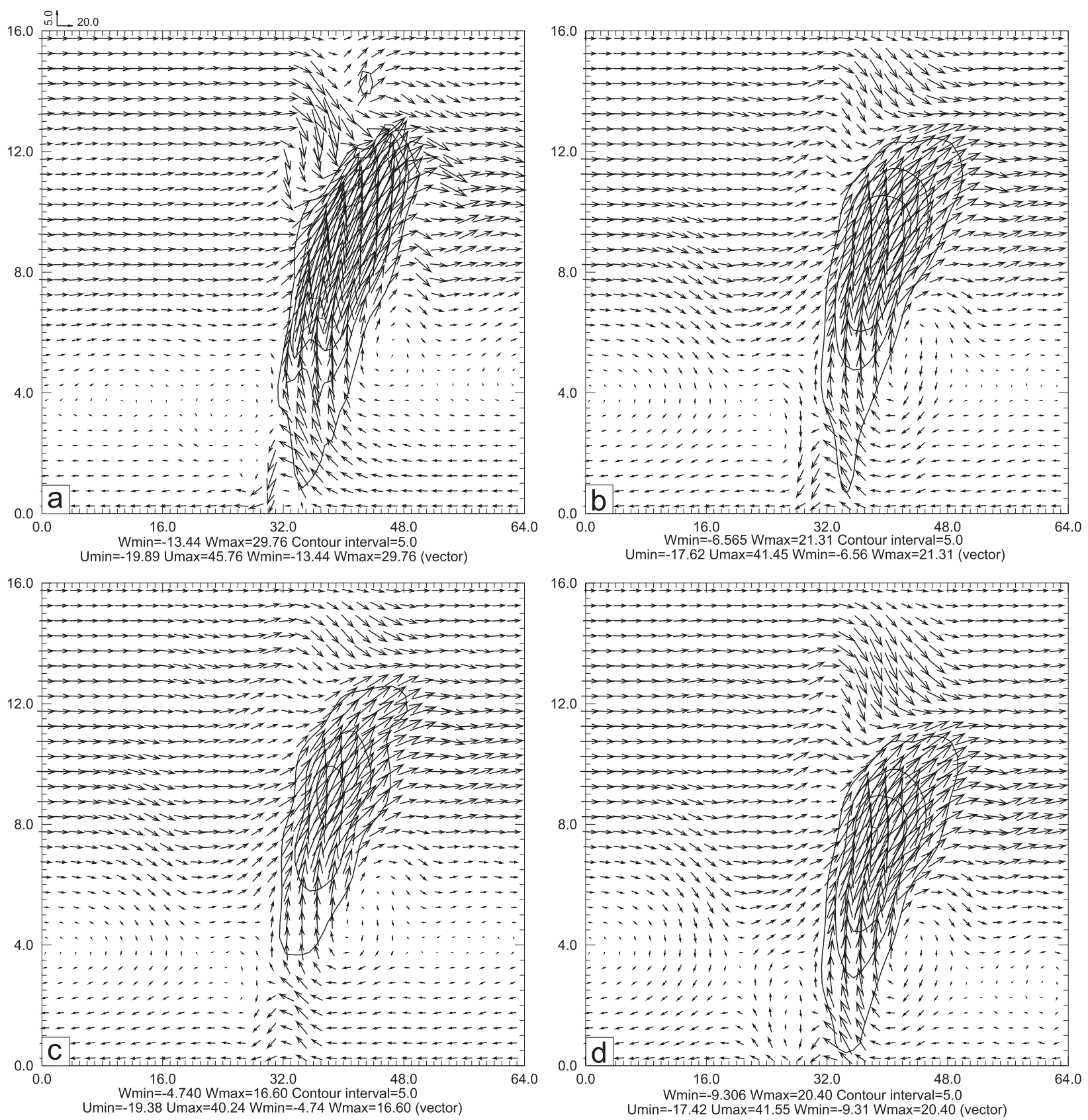

FIG. 5. As in Fig. 2, but for (a) truth simulation, (b) CNTL1_150, (c) NoBB1_150, and (d) NoCV1_150.

exhibits an additional $7.1 \mathrm{~m} \mathrm{~s}^{-1}$ RMS error of horizontal winds compared to CNTL1 experiment at the range of $220 \mathrm{~km}$ (Fig. 3a). The RMS error differences for vertical velocities between CNTL1 and NoCV1 experiments are evident when the surface range is over $150 \mathrm{~km}$ (Fig. 3b). Therefore, in the sense of the evolution of RMS errors, we can conclude that overlooking the earth curvature has a much greater negative impact on variational wind analysis than the neglect of beam broadening.
As the RMS statistics suggest, the differences in the 3D wind fields among all three categories of experiments, CNTL1, NoBB1 and NoCV1, should be very small when the distance between the storm and radars is less than $60 \mathrm{~km}$. Figures 1 and 2 confirm this conclusion. Figure 1 shows that the horizontal wind and vertical velocity fields at $3.5 \mathrm{~km}$ AGL for the truth simulation and the three experiments, CNTL1_60, NoBB1_60, and NoCV1_60, where the radar is $60 \mathrm{~km}$ from the storm. Though the 3DVAR analysis is not perfect, the horizontal 
cyclonic rotation associated with the right and left movers are clearly evident in all three experiments (Figs. 1b-d). They are all close to the truth simulation (Fig. 1a). The analyzed maximum vertical velocities (Figs. $2 \mathrm{~b}-\mathrm{d}$ ) for all three categories of experiments are generally several meters per second weaker than the truth simulation, but the pattern is nearly the same for all three experiments. So, the error from neglecting both beam broadening and earth curvature at this range is small.

When the distance between the storm and radar location is $150 \mathrm{~km}$ or greater, the differences among these experiments become larger and can no longer be ignored. As an example, horizontal cross sections at $z=$ $3.5 \mathrm{~km}$ and vertical cross sections are plotted in Figs. 4 and 5 for the surface range of $150 \mathrm{~km}$ (the truth simulation is replotted for ease of comparison). It is clearly evident that the rotation signature near the center of the storm in Fig. 4b for CNTL1_150 is stronger than that in Fig. 4c for NoBB1_150. In addition, Fig. 5b shows a much stronger and deeper rotation updraft than Fig. 5c. The maximum vertical velocity in Fig. $5 \mathrm{~b}$ is $21.31 \mathrm{~m} \mathrm{~s}^{-1}$, much closer to the simulation result (as shown in Fig. 5a) than that in Fig. 5b, which is only $16.60 \mathrm{~m} \mathrm{~s}^{-1}$. Apparently, CNTL1_150 experiment does a better job for the wind analysis than NoBB1_150, in which no effect of beam broadening is considered.

For experiment NoCV1_150, in which the influence of the earth's curvature is not considered, Fig. $4 \mathrm{~d}$ shows that the perturbation horizontal winds are unexpectedly strong and quite noisy. The signatures of cyclonic rotation within each of the cells are not so well analyzed. Although the strength of the major updraft in Fig. 5d is well captured, just as in Fig. 5b of CNTL1_150, the updraft in Fig. 5d is incorrectly positioned in the vertical direction, about $1 \mathrm{~km}$ below than that in Fig. 5a. All these distorted features are evidently caused by the neglect of the effect of the earth curvature in the radar forward observation operator. It should be noted that the wind analysis generally becomes worse even in CNTL1_150 experiment because of the poorer resolution in the data at that distance.

It is demonstrated that the impacts of both the beam broadening and earth curvature are dependent on the surface range between the center of the storm and the radar location. It appears that, within a range of $60 \mathrm{~km}$, both the impacts of beam broadening and earth curvature can be neglected. As the distance increases beyond $60 \mathrm{~km}$, more and more additional errors are introduced into the wind analysis from both earth curvature and beam broadening effects. Specifically, the neglect of the earth curvature exhibits much more negative impact than the neglect of the beam broadening. When the distance to the storm exceeds $150 \mathrm{~km}$, overlooking the earth curvature

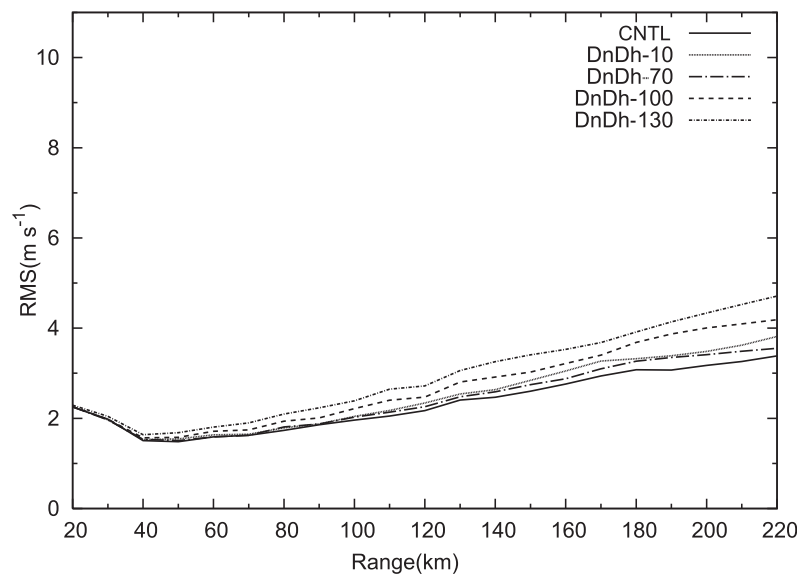

FIG. 6. The variation of RMS errors with the distance between the center of the storm and radar locations for horizontal wind. The solid lines are for CNTL1 experiments, the dotted lines are for DnDh-10 experiments, the dot-dashed lines are for DnDh-70 experiments, the dashed lines are for DnDh-100 experiments, and the short dotted-dashed lines are for the DnDh-130 experiments.

and the beam broadening will both bring much more obvious negative impact on the three-dimensional wind analysis. So, the Cartesian ray path equation and a simple interpolation are not recommended when the distance to the storm is greater than $150 \mathrm{~km}$.

The four-thirds earth radius model assumes that $d n / d h$ equals $-1 /(4 a)$, about $-39.2 \times 10^{-6} \mathrm{~km}^{-1}$, for the standard atmosphere. However, $d n / d h$ can deviate from this value more than $100 \times 10^{-6} \mathrm{~km}^{-1}$ in storm-favoring environments (Gao06). The impact of a refractivity gradient with different $d n / d h$ is investigated now through four categories of data analysis experiments as described in the last section. The RMS error of horizontal wind for the experiments CNTL1, DnDh-10, DnDh-70, DnDh-100, and DnDh-130 are plotted in Fig. 6. It is shown that the impact of using different values of $d n / d h$ instead of $-1 /(4 a)$ for standard atmosphere is rather small. The additional RMS error due to the use of the fourth-thirds earth radius model is generally less than $0.42 \mathrm{~m} \mathrm{~s}^{-1}$ within a range of $100 \mathrm{~km}$. The impact will gradually increase as the range increases. When the radar is very far from the storm (beyond $190 \mathrm{~km}$ ) and the absolute value of $d n / d h$ is very large (more than $130 \times 10^{-6} \mathrm{~km}^{-1}$ ), the additional RMS error is over $1 \mathrm{~m} \mathrm{~s}^{-1}$. The additional RMS errors of vertical velocity (not shown) introduced by the use of $d n / d h=-1 /(4 a)$ are all less than $0.2 \mathrm{~m} \mathrm{~s}^{-1}$. The 3D wind plots (not shown) also confirmed the above statements. It is concluded that the impact of refractive index can be neglected for most applications. Because the impact is so small, this effect will not be discussed in the following intermittent data assimilation experiments. 


\section{b. The impact on radar data assimilation cycles}

To investigate how the errors introduced by neglecting the beam broadening and the earth curvature are accumulated during an intermittent data assimilation and to investigate how the retrievals of other model variables, such as potential temperature and moisture, are impacted, two time series of data assimilation with 5-min intervals are performed during a 1-h-long data assimilation period. As discussed in section 4, the first three intermittent data assimilation experiments referred to as CNTLM_60, NoBBM_60, and NoCVM_60 are conducted using data sampled from $t=120 \mathrm{~min}$ to $t=180 \mathrm{~min}$ of model simulation with a radar distance of $60 \mathrm{~km}$ when the data assimilation experiments begin. Three more experiments CNTLM_150, NoBBM_150, and NoCVM_150 are conducted for the radar distance of $150 \mathrm{~km}$ at the beginning of data assimilation. The results from these six experiments are discussed below.

Figure 7 shows the horizontal winds, perturbation potential temperature, and reflectivity at $250 \mathrm{~m}$ AGL (first model level above surface), and Fig. 8 shows the horizontal wind and vertical velocity fields at $3.5 \mathrm{~km}$ AGL, at 140,155 , and $170 \mathrm{~min}$ of model time. Recall that the model assimilation begins at $t=120 \mathrm{~min}$. They are shown for the truth simulation, cycled 3DVAR assimilation for experiments CNTLM_60, NoBBM_60, and No_CVM_60, as described above. For all three experiments, Figs. 7d,g,j show that, after four cycles at $t=140 \mathrm{~min}$, the assimilation has retrieved some weak potential temperature perturbations. Though no reflectivity is assimilated, the model established the reflectivity pattern quite similar to the truth simulation, although covering a smaller area after $20 \mathrm{~min}$ of assimilation. A small positive temperature perturbation is found where there should be cooling (Figs. 7d,g,j). At the 3.5-km level (Figs. 8d,g,j), an updraft is established well at the correct location, and its strength and structure are quite similar to the truth (Fig. 8a). After three more analysis cycles at $t=155 \mathrm{~min}$, the low-level flow immediately underneath the storm cells becomes closer to the truth (Figs. 7e,h,k versus Fig. 7b), but the area of outflow and cold pool on the southwest side remain smaller than the truth. At the $3.5-\mathrm{km}$ level, the perturbation horizontal winds and the updrafts are well captured in all three experiments by $t=155$ min (Figs. 8e,h,k versus Fig. 8b).

By $t=170 \mathrm{~min}$, the analysis is further improved. In fact, by this time, there are no significant differences from the truth in either the low-level or midlevel fields (Figs. 7f,i,m, $8 \mathrm{f}, \mathrm{i}, \mathrm{m})$. General storm structures including the precipitation pattern are well retrieved during this 1-h data assimilation in all three experiments, though the results from NoCVM_60 are not quite as good. This reinforces that the impacts of beam broadening and earth curvature on radar data assimilation cycles for retrieving other model variables from the radial wind of two radars are generally small when the storm is not far from two radars.

Although the RMS error is generally not well suited as a verification metric for storm-scale phenomena, we use it here for comparison among different experiments and also visually compare plotted fields to verify the result The RMS errors for several analyzed fields are shown in Fig. 9. The RMS errors for $V_{h}$ components decrease with time but very slowly. The variations of RMS errors for $w$ are not stable, possibly because of small phase or position errors. The RMS errors for $\theta^{\prime}$ decrease for the first $40 \mathrm{~min}$ of assimilation, then increase with time again. Only the errors for $q_{v}$ decrease nearly monotonically with time. The $q_{v}$ RMS error is reduced to $0.28 \mathrm{~g} \mathrm{~kg}^{-1}$ in CNTLM_60 and to 0.31 and $0.35 \mathrm{~g} \mathrm{~kg}^{-1}$ in NoBBM_60 and NoCVM_60, respectively. Figure 9 generally shows that the RMS errors of $V_{h}, w, \theta^{\prime}$, and $q_{v}$ stay very close for all three experiments, though NoCVM_60 has slightly larger errors in $V_{h}$. The RMS errors again suggest that the effect of beam broadening and earth curvature is generally small when the storm is not far from radar.

We now turn to the results for experiments CNTLM_150, NoBBM_150, and NoCVM_150. Figures 10 and 11 show that, in general, the results are significantly worse in all three experiments than the prior $60-\mathrm{km}$ experiments. The overall storm structures are poorly resolved compared to CNTLM_60, NoBBM_60, and NoCVM_60. However, among the three experiments for the range of $150 \mathrm{~km}$, the overall structure of the storm for CNTLM 150 is the best and quite similar to those of the truth toward the end of the assimilation.

In experiment NoBBM_150, the precipitation area is pretty small and the cold pool is very weak at $140 \mathrm{~min}$ (i.e., after $20 \mathrm{~min}$ of assimilation; Fig. $10 \mathrm{~g}$ ), but the pattern of horizontal winds and strength of updraft at the $3.5-\mathrm{km}$ level is similar to the truth (Fig. $11 \mathrm{~g}$ versus Fig. 11a). At $155 \mathrm{~min}$, the analysis looks better, but both the horizontal wind and vertical velocity field look noisy, and there exist several small centers for positive or negative contours that are not supported by the truth simulation (Fig. 11h versus Fig. 11b). At the end of the assimilation (Figs. 10i, 11i), the reflectivity and updraft patterns look much closer to the truth. Clearly, at this very large radar distance, the neglect of beam broadening worsens the assimilation results. However, the impact is limited and the internal structures of thunderstorms can still be obtained well by the end of $1 \mathrm{~h}$ of assimilation.

When the effect of earth curvature is not considered at the range of $150 \mathrm{~km}$, the analyzed low-level cold pool, gust front, and precipitation pattern differ markedly from those of the truth (Figs. 10j,k,m versus Figs. 10a-c) and from the control assimilation at the same radar distance (versus 

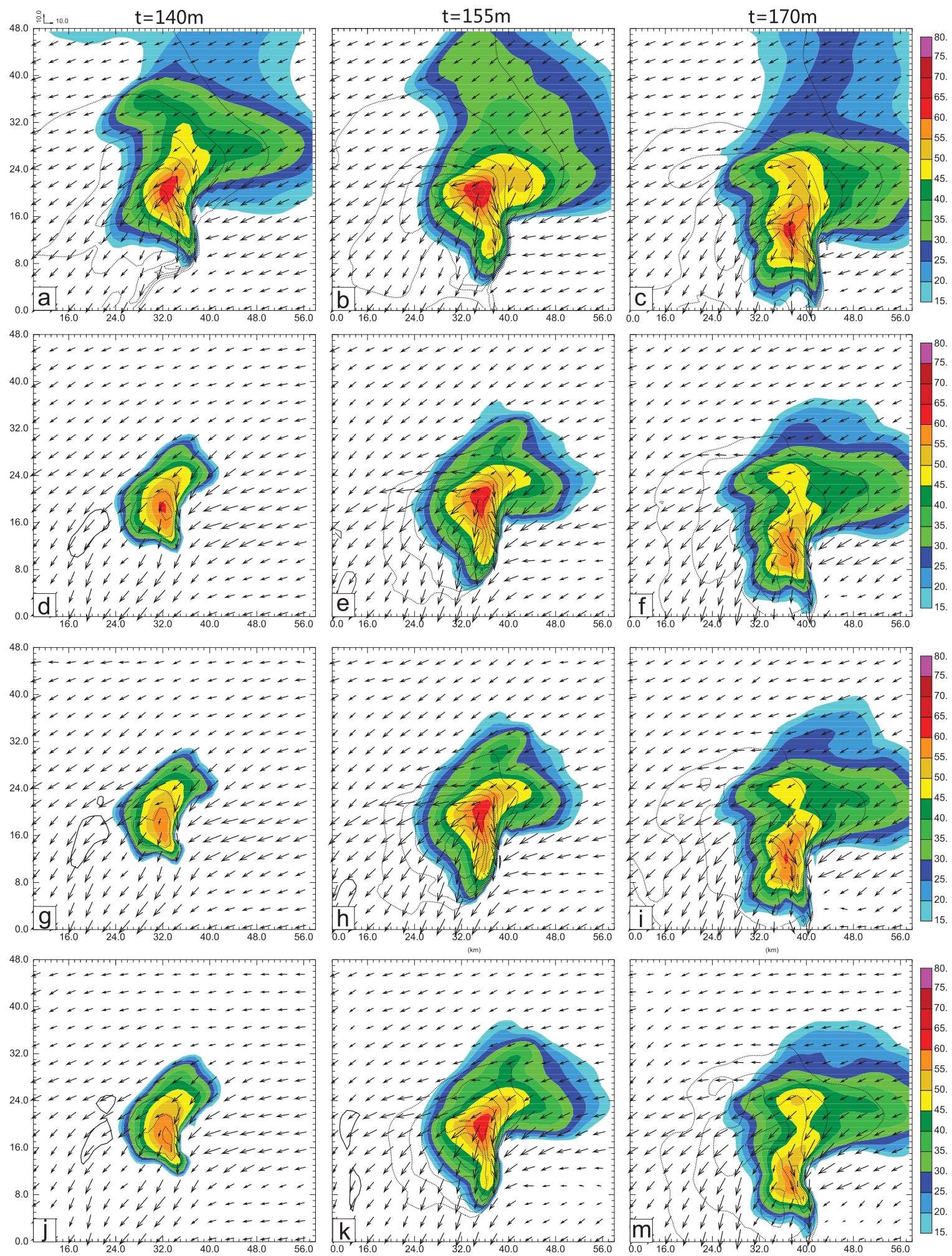

FIG. 7. The total $u-v$ wind vector, perturbation potential temperature (contour at every $1 \mathrm{~K}$ ), and reflectivity (colored) at $z=250 \mathrm{~m}$ AGL and (left)-(right) $t=140,155$, and $170 \mathrm{~min}$ for (a)-(c) truth simulation, (d)-(f) CNTLM_60,(g)-(i) NoBBM_60, and (j)-(m) NoCVM_60: solid contour for positive and dashed contour for negative. 

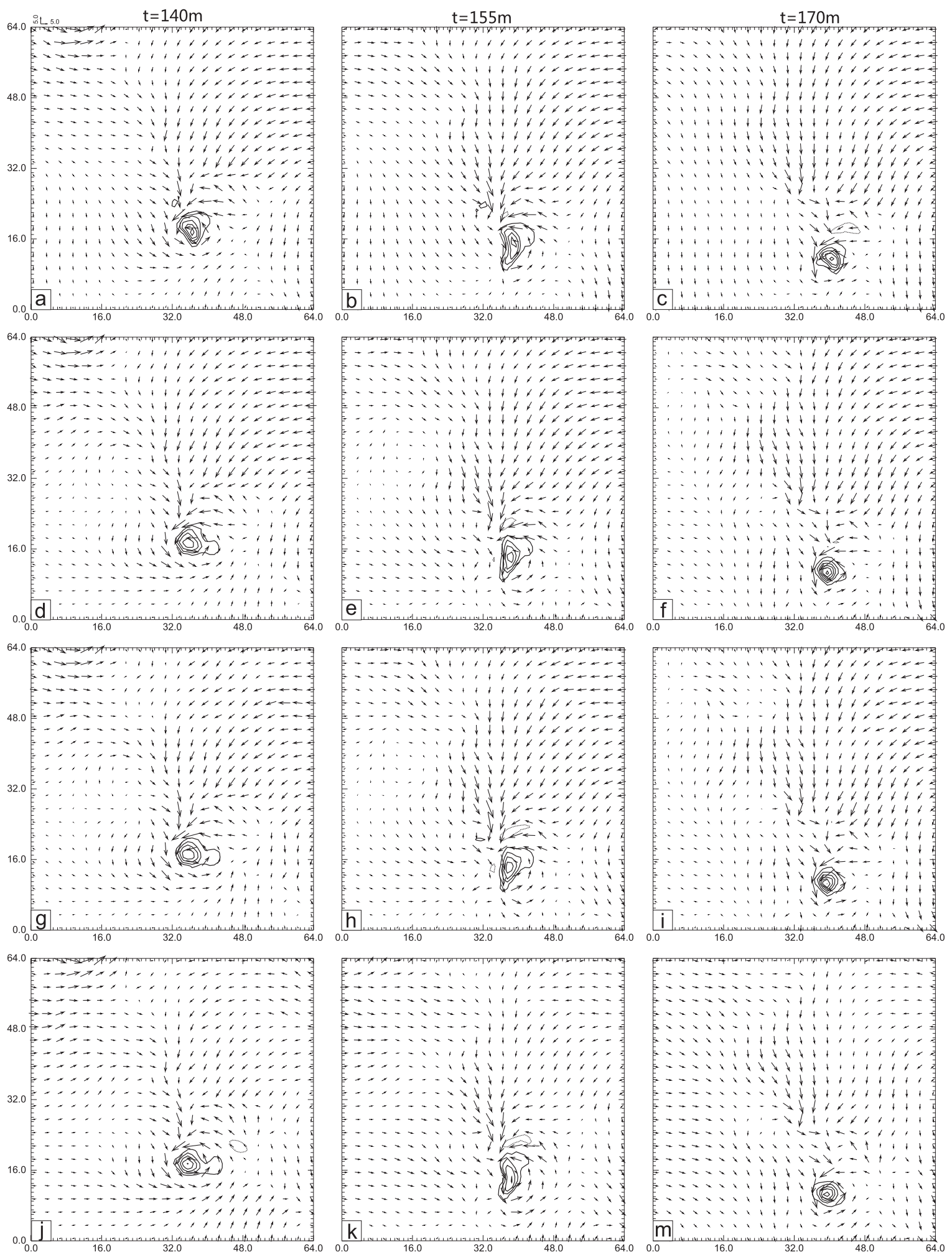

FIG. 8. The perturbation $u-v$ wind vector and vertical velocity (contour at every $5 \mathrm{~m} \mathrm{~s}^{-1}$ ) at $z=3.5 \mathrm{~km} \mathrm{AGL} \mathrm{and} \mathrm{(left)-(right)} t=140$, 155, and 170 min for (a)-(c) truth simulation, (d)-(f) CNTLM_60, (g)-(i) NoBBM_60, and (j)-(m) NoCVM_60: solid contour for positive and dashed contour for negative. 

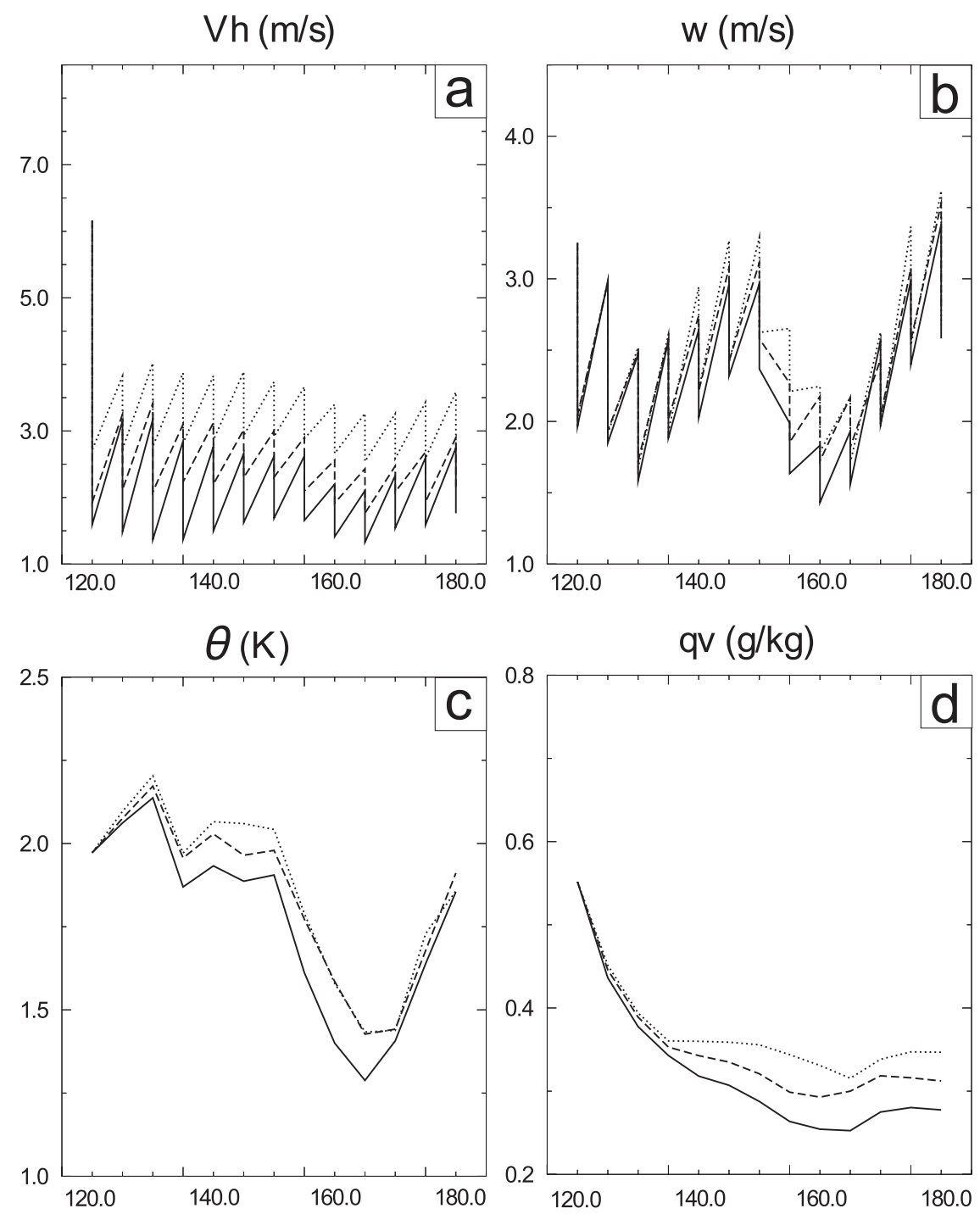

FIG. 9. The evolution of the RMS errors with time for different model variables. The solid lines are for CNTLM_60, the dashed lines are for NoBBM_60, and the dotted lines are for NoCVM_60.

Figs. 10d-f). At $t=155$ min, the midlevel updraft appears broader and the pattern of horizontal flow is significantly different from the truth. At $t=170 \mathrm{~min}$, the reflectivity core becomes distorted and the hook echo is poorly defined after $50 \mathrm{~min}$ of assimilation (Fig. $10 \mathrm{~m}$ versus Fig. 10c). Also at this time, there are a few spurious updrafts within the analysis domain (Fig. $11 \mathrm{~m}$ ). Overall, the analysis is significantly worsened when the effect of earth curvature is not considered at a radar distance of $150 \mathrm{~km}$.

The variations of the RMS error in horizontal wind components $V_{h}$, vertical velocity $w$, perturbation potential temperature $\theta$, and perturbation water vapor mixing ratio $q_{v}$ are plotted in Fig. 12. It is demonstrated that the RMS errors in NoBBM_150 are generally larger than those in CNTLM_150 but do not deviate much. The NoCVM_150 experiment yields the worst results with the largest RMS errors during the 1-h-long assimilation period among all three experiments, especially for variables $V_{h}$ and $q_{v}$. These error statistics also indicate that when a storm is $150 \mathrm{~km}$ from the radar, neglecting beam broadening worsens the results slightly, whereas overlooking earth curvature produces significantly worse results for retrieved model variables.

\section{Summary and discussion}

To utilize high-resolution radar radial velocity data in storm-scale data assimilation, it is necessary to compute 

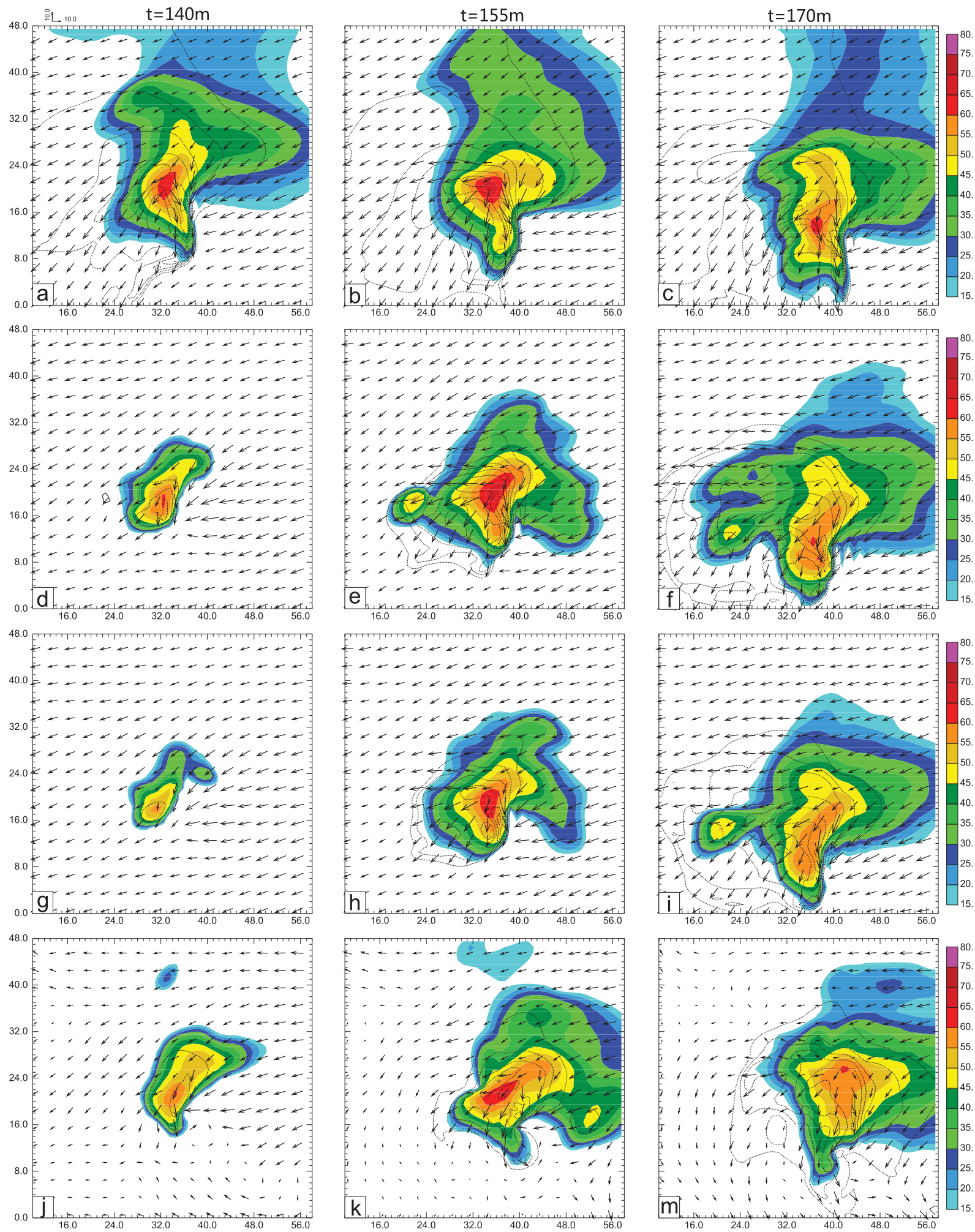

FIG. 10. As in Fig. 7, but for (a)-(c) truth simulation, (d)-(f) CNTLM_150, (g)-(i) NoBBM_150, and (j)-(m) NoCVM_150: solid contour for positive and dashed contour for negative. 

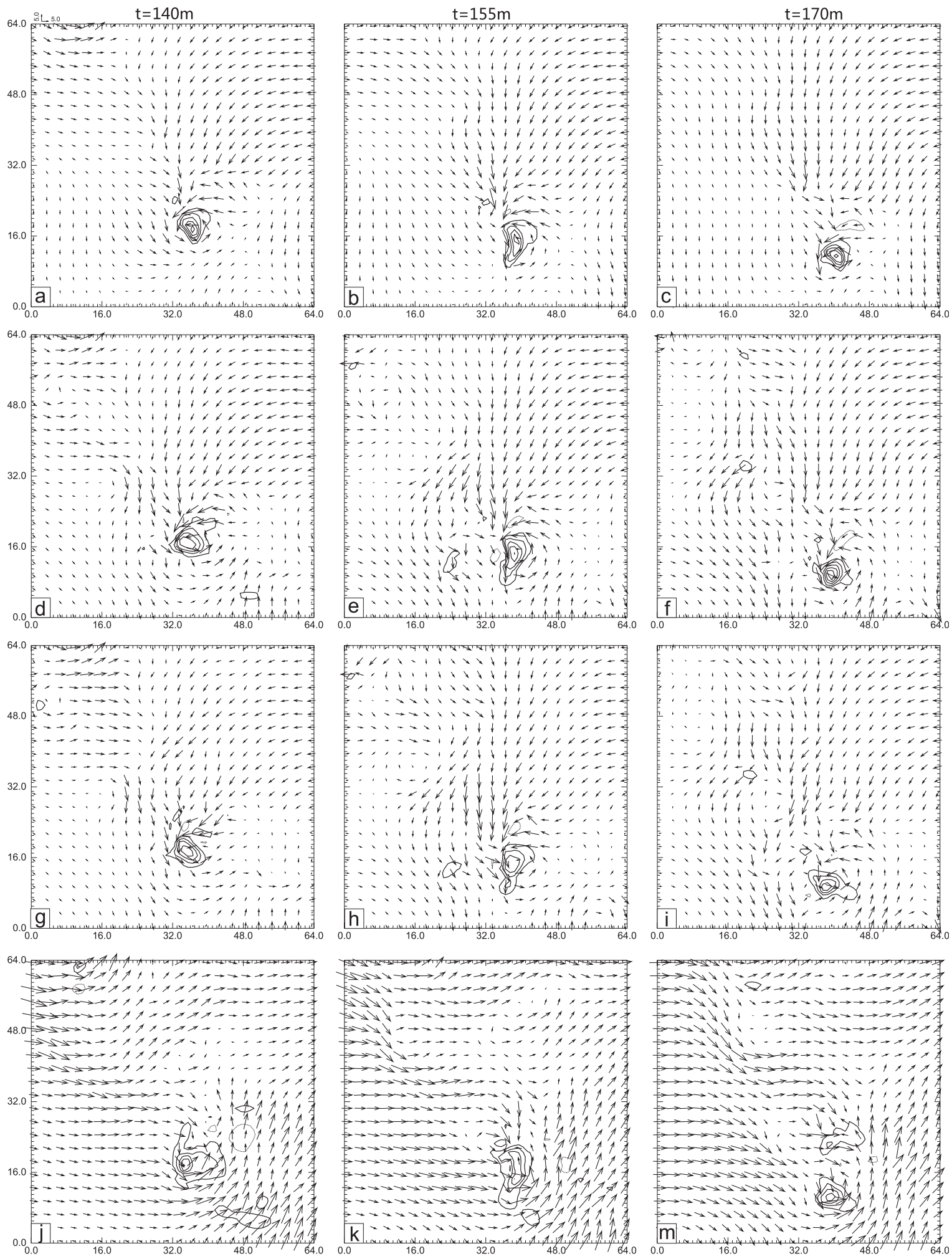

FIG. 11. As in Fig. 8, but for (a)-(c) truth simulation, (d)-(f) CNTLM_150, (g)-(i) NoBBM_150, and (j)-(m) NoCVM_150: solid contour for positive and dashed contour for negative. 

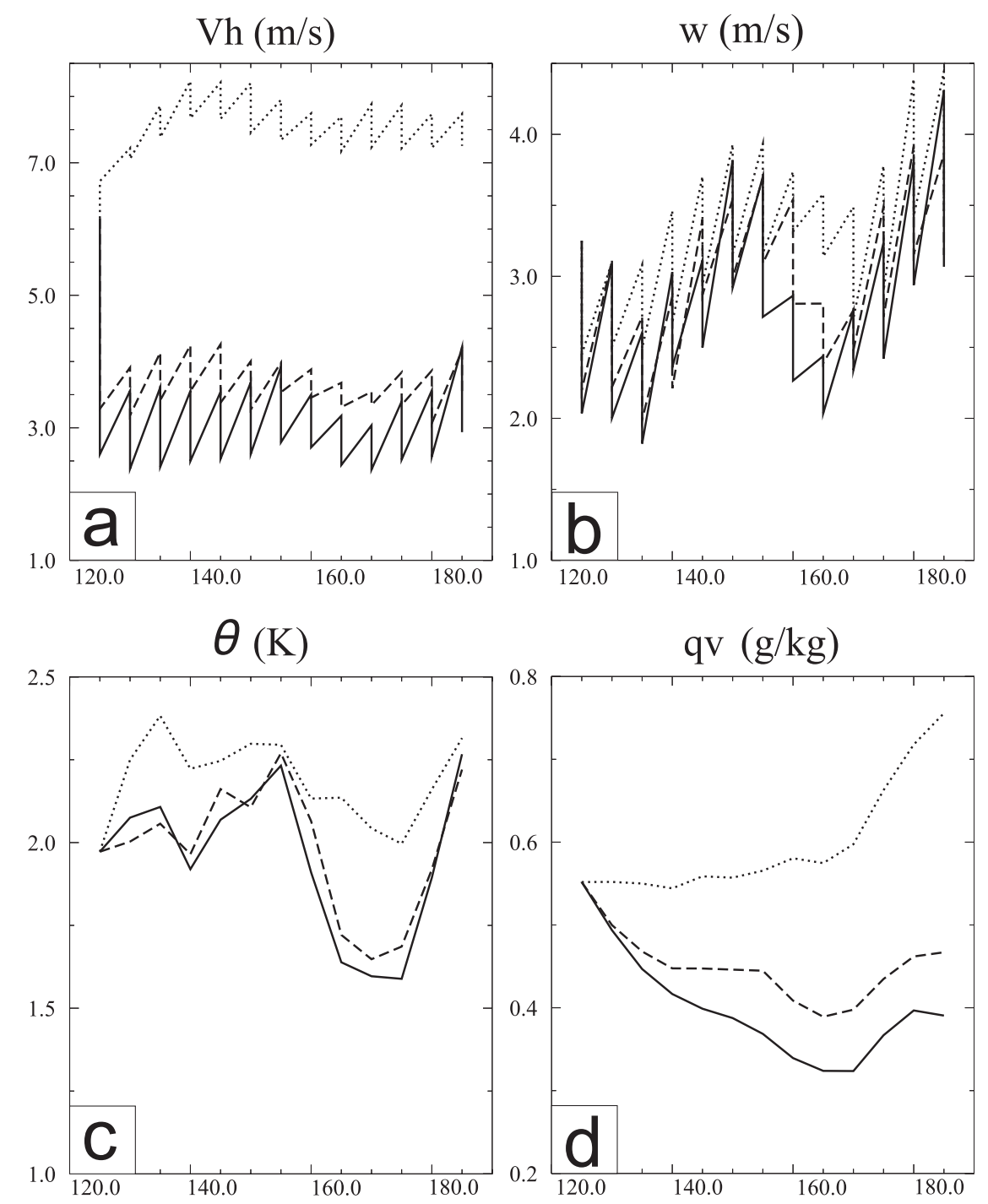

FIG. 12. The evolution of the RMS error for different model variables. The solid lines are for CNTLM_150, the dashed lines are for NoBBM_150, and the dotted lines are for NoCVM_150.

the model counterpart of radial winds by converting $u, v$, and $w$ winds on model grids into radial velocity in radar coordinates. This is called the radar forward observation operator. The most accurate forward observation operator includes considering the effect of beam broadening and the earth curvature. However, this may lead to higher computational cost that could impact the lead time of a forecast system or require additional computational resources. So, some past research used a very simple form of radar observation operator by neglecting the two effects mentioned above, or where better formulations were used, but the impact of that choice was not explicitly measured. In this study, we examined the effects of these assumptions on assimilating data from an idealized simulated supercell storm. It is shown that both the effects of beam broadening and earth curvature can only be neglected when the radar is near the storm, within $60 \mathrm{~km}$, as demonstrated by this study.

For wind analysis at a single time, as the surface range increases, more error will be introduced into the analysis by the neglect of the two effects. The effect of beam broadening becomes evident and can cause relatively large errors for ranges at and beyond $150 \mathrm{~km}$. The effect of earth curvature is very significant when the surface range is beyond $60 \mathrm{~km}$ because of vertical location errors in the data. The impact of refractive index gradient is also tested. It is shown that the variation of refractive index gradient has a very small impact on the wind analysis. It is acknowledged that there are extreme cases where ducting and other effects can occur, 
causing false echoes and ground or sea clutter, but it is assumed such data will be properly screened from use in data assimilation.

In two series of 1-h-long data assimilation experiments, it is shown that the impact of both effects is not significant for retrieving all model variables when the radars are relatively close to the storm (generally within $60 \mathrm{~km}$ ). When the radars are far from the storm, not accounting for beam broadening has a rather small effect on the accuracy of assimilation results after 1-h assimilation. So, the effect of beam broadening can be generally overlooked in radar data assimilation. On the other hand, ignoring the earth's curvature leads to significant errors (especially beyond $150 \mathrm{~km}$ ) for retrieved model variables and reflectivity because of vertical location error in the data. The results of this study may provide useful guidance for application of radar radial velocity data to storm-scale diagnostic studies as well as numerical weather prediction.

Acknowledgments. This research was supported by NSF Grants ATM-0738370, EEC-031347, ATM-0608168, ATM-0802888, and ATM-0530814. The second and fourth authors were also partial supported by China NSF under Grant 40620120437. Computations were performed at the Pittsburgh Supercomputing Center (PSC) and Oklahoma Supercomputing Center for Research and Education (OSCER). Thanks for the comments from the anonymous reviewers to improve this paper from its original form.

\section{REFERENCES}

Bean, B. R., and E. J. Dutton, 1968: Radio Meteorology. Dover, 435 pp.

Brewster, K. A., 2003: Phase-correcting data assimilation and application to storm-scale numerical weather prediction. Part II: Application to a severe storm outbreak. Mon. Wea. Rev., 131, 493-507.

Caumont, O., and V. Ducrocq, 2008: What should be considered when simulating Doppler velocities measured by groundbased weather radars? J. Appl. Meteor. Climatol., 47, 22562262.

Crum, T. D., and R. L. Alberty, 1993: The WSR-88D and the WSR88D Operational Support Facility. Bull. Amer. Meteor. Soc., 74, 1669-1687.

_ - R. E. Saffle, and J. W. Wilson, 1998: An update on the NEXRAD program and future WSR-88D support to operations. Wea. Forecasting, 13, 253-262.

Doviak, R. J., and D. S. Zrnić, 1993: Doppler Radar and Weather Observations. 2nd ed. Academic Press, 562 pp.

Droegemeier, K. K., 1990: Toward a science of storm-scale prediction. Preprints, 16th Conf. on Severe Local Storms, Alberta, Canada, Amer. Meteor. Soc., 256-262.

- 1997: The numerical prediction of thunderstorms: Challenges, potential benefits, and results from real time operational tests. WMO Bull., 46, 324-336.
Gao, J., M. Xue, Z. Wang, and K. K. Droegemeier, 1998: The initial condition and explicit prediction of convection using ARPS forward assimilation and adjoint methods with WSR-88D data. Preprints, 12th Conf. on Numerical Weather Prediction, Phoenix, AZ, Amer. Meteor. Soc., 176-178.

, - A A. Shapiro, and K. K. Droegemeier, 1999: A variational method for the analysis of three-dimensional wind fields from two Doppler radars. Mon. Wea. Rev., 127, 2128-2142.

- — - K. Brewster, F. Carr, and K. K. Droegemeier, 2002: New development of a 3DVAR system for a nonhydrostatic NWP model. Preprints, 19th Conf. on Weather Analysis and Forecasting/15th Conf. on Numerical Weather Prediction, San Antonio, TX, Amer. Meteor. Soc., 12.4. [Available online at http://ams.confex.com/ams/pdfpapers/47480.pdf.]

- $-\longrightarrow$, and K. K. Droegemeier, 2004: A threedimensional variational data assimilation method with recursive filter for single-Doppler radar. J. Atmos. Oceanic Technol., 21, 457-469.

— K. Brewster, and M. Xue, 2006: A comparison of the radar ray path equations and approximations for use in radar data assimilation. Adv. Atmos. Sci., 23, 190-198, doi:10.1007/ s00376-006-0190-3.

Ge, G., and J. Gao, 2007: Latest development of 3DVAR system for ARPS and its application to a tornadic supercell storm. Preprints, 22nd Conf. on Weather Analysis and Forecasting/ 18th Conf. on Numerical Weather Prediction, Park City, Utah, Amer. Meteor. Soc., 2B.6. [Available online at http://ams. confex.com/ams/pdfpapers/124724.pdf.]

Gu, W., H. Gu, and Q. Xu, 2001: Impact of single-Doppler radar observations on numerical prediction of 7 May 1995 Oklahoma squall line. Preprints, Fifth Symp. on Integrated Observing Systems, Albuquerque, NM, Amer. Meteor. Soc., 139-142.

Hodur, R. M., 1997: The Naval Research Laboratory's Coupled Ocean/Atmosphere Mesoscale Prediction System (COAMPS). Mon. Wea. Rev., 125, 1414-1430.

$\mathrm{Hu}$, M., M. Xue, and K. Brewster, 2006a: 3DVAR and cloud analysis with WSR-88D level-II data for the prediction of Fort Worth tornadic thunderstorms. Part I: Cloud analysis and its impact. Mon. Wea. Rev., 134, 675-698.

,-- J. Gao, and K. Brewster, 2006b: 3DVAR and cloud analysis with WSR-88D level-II data for the prediction of Fort Worth tornadic thunderstorms. Part II: Impact of radial velocity analysis via 3DVAR. Mon. Wea. Rev., 134, 699-721.

Klemp, J. B., and R. Rotunno, 1983: A study of the tornadic region within a supercell thunderstorm. J. Atmos. Sci., 40, 359-377.

, R. B. Wilhelmson, and P. S. Ray, 1981: Observed and numerically simulated structure of a mature supercell thunderstorm. J. Atmos. Sci., 38, 1558-1580.

Lilly, D. K., 1990: Numerical prediction of thunderstorms: Has its time come? Quart. J. Roy. Meteor. Soc., 116, 779-798.

Lin, Y., R. D. Farley, and H. D. Orville, 1983: Bulk parameterization of the snow field in a cloud model. J. Appl. Meteor., 22, $1065-1092$.

Probert-Jones, J. R., 1962: The radar equation in meteorology. Quart. J. Roy. Meteor. Soc., 88, 485-495.

Purser, R. J., W. Wu, D. F. Parrish, and N. M. Roberts, 2003a: Numerical aspects of the application of recursive filters to variational statistical analysis. Part I: Spatially homogeneous and isotropic Gaussian covariances. Mon. Wea. Rev., 131, 1524-1535.

,,$-- \ldots$, and $\longrightarrow, 2003 \mathrm{~b}$ : Numerical aspects of the application of recursive filters to variational statistical analysis. 
Part II: Spatially inhomogeneous and anisotropic general covariances. Mon. Wea. Rev., 131, 1536-1548.

Qiu, C., and Q. Xu, 1992: A simple adjoint method of wind analysis for single-Doppler data. J. Atmos. Oceanic Technol., 9, $588-598$.

_ , and — 1996: Least squares retrieval of microburst winds from single-Doppler radar data. Mon. Wea. Rev., 124, 11321144.

Ray, P. S., B. C. Johnson, K. W. Johnson, J. S. Bradberry, J. J. Stephens, K. K. Wagner, R. B. Wilhelmson, and J. B. Klemp, 1981: The morphology of several tornadic storms on 20 May 1977. J. Atmos. Sci., 38, 1643-1663.

Rihan, F. A., C. G. Collier, S. P. Ballard, and S. J. Swarbrick, 2008: Assimilation of Doppler radial winds into a 3D-Var system: Errors and impact of radial velocities on the variational analysis and model forecasts. Quart. J. Roy. Meteor. Soc., 134, $1701-1716$

Salonen, K., 2002: Observation operator for Doppler radar radial winds in HiRLAM 3D-Var. ERAD Publication Series, No. 1, 405-408.

Serafin, R. J., and J. W. Wilson, 2000: Operational weather radar in the United States: Progress and opportunity. Bull. Amer. Meteor. Soc., 81, 501-518.

Shapiro, A., P. Robinson, J. Wurman, and J. Gao, 2003: SingleDoppler velocity retrieval with rapid scan radar data. J. Atmos. Oceanic Technol., 20, 1758-1775.

Sun, J., and N. A. Crook, 1997: Dynamical and microphysical retrieval from Doppler radar observations using a cloud model and its adjoint. Part I: Model development and simulated data experiments. J. Atmos. Sci., 54, 1642-1661.

$\longrightarrow$, and — 1998: Dynamical and microphysical retrieval from Doppler radar observations using a cloud model and its adjoint. Part II: Retrieval experiments of an observed Florida convective storm. J. Atmos. Sci., 55, 835-852.

, and - 2001: Real-time low-level wind and temperature analysis using single WSR-88D data. Wea. Forecasting, 16, 117-132.

D. W. Flicker, and D. K. Lilly, 1991: Recovery of threedimensional wind and temperature fields from simulated single-Doppler radar data. J. Atmos. Sci., 48, 876-890.
Tong, M., 2006: Ensemble Kalman filter assimilation of Doppler radar data for the initialization and prediction of convective storms. Ph.D dissertation, University of Oklahoma, $263 \mathrm{pp}$.

Weygandt, S. S., A. Shapiro, and K. K. Droegemeier, 2002a: Retrieval of initial forecast fields from single-Doppler observations of a supercell thunderstorm. Part I: Single-Doppler velocity retrieval. Mon. Wea. Rev., 130, 433-453.

$\longrightarrow, \ldots$, and $\longrightarrow, 2002 \mathrm{~b}$ : Retrieval of initial forecast fields from single-Doppler observations of a supercell thunderstorm. Part II: Thermodynamic retrieval and numerical prediction. Mon. Wea. Rev., 130, 454-476.

Wood, V. T., and R. A. Brown, 1997: Effects of radar sampling on single-Doppler velocity signatures of mesocyclones and tornadoes. Wea. Forecasting, 12, 928-938.

Xu, Q., C. Qiu, H. D. Gu, and J. Yu, 1995: Simple adjoint retrievals of microburst winds from single-Doppler radar data. Mon. Wea. Rev., 123, 1822-1833.

—, H. Gu, and S. Yang, 2001: Simple adjoint method for threedimensional wind retrievals from single-Doppler radar. Quart. J. Roy. Meteor. Soc., 127, 1053-1067.

Xue, M., K. K. Droegemeier, and V. Wong, 2000: The Advanced Regional Prediction System (ARPS) - A multiscale nonhydrostatic atmospheric simulation and prediction tool. Part I: Model dynamics and verification. Meteor. Atmos. Phys., 75, 161-193.

— , and Coauthors, 2001: The Advanced Regional Prediction System (ARPS) - A multi-scale nonhydrostatic atmospheric simulation and prediction tool. Part II: Model physics and applications. Meteor. Atmos. Phys., 76, 143-166.

_, D.-H. Wang, J.-D. Gao, K. Brewster, and K. K. Droegemeier, 2003: The Advanced Regional Prediction System (ARPS), storm-scale numerical weather prediction and data assimilation. Meteor. Atmos. Phys., 82, 139-170.

_, M. Tong, and K. K. Droegemeier, 2006: An OSSE framework based on the ensemble square root Kalman filter for evaluating the impact of data from radar networks on thunderstorm analysis and forecasting. J. Atmos. Oceanic Technol., 23, 46-66.

Zhao, Q., J. Cook, Q. Xu, and P. Harasti, 2006: Using radar wind observations to improve mesoscale numerical weather prediction. Wea. Forecasting, 21, 502-522. 